\title{
DESIGN OF FULLY AUTOMATIC CALIBRATION MACHINE FOR REFERENCE BLOCK BASED ON MACHINE VISION
}

\author{
Xiang Ren ${ }^{1}$, Jin $\mathrm{Yu} \mathrm{Lu}{ }^{2}$, Zecheng $\mathrm{Tao}^{3}$ \\ ${ }^{1}$ Metrology of He nan Institute, Zhengzhou, China, 243553773@qq.com \\ ${ }^{2}$ Metrology of He nan Institute, Zhengzhou, China, jinyu lu@ 126.com \\ ${ }^{3}$ Innovative Testing Technology Co., Ltd., Kunshan, China, tzc@cxtest.com
}

\begin{abstract}
:
In order to solve the problems of the existing standard Brinell hardness machine such as low automation, the time-consuming indentation measurement and the human influence in indentation measurement, a calibration machine for reference block was designed. It shows that this machine can reduce the influence of the temperature difference between the hardness block and the measurement platform; it eliminates the time for the indentation measuring system to find the indentation and juxtapose it with the centre of the field of view and implements fully Brinell hardness automatic calibration.
\end{abstract}

Keywords: automatic indentation measuring system; machine vision technology

\section{INTRODUCTION}

The Brinell hardness test method is the simplest and fastest method in the mechanical property test of materials. It is one of the main methods for determining the mechanical properties of materials, inspecting product quality, determining reasonable heat treatment specifications and machining processes [1]. As a standard calibration machine which must have the function of reproducing and storing the unit value, and its reliability has special significance for the stable measurement performance which required by the standard device [2]. Among the existing reference block calibration machines, they basically adopts the loading method of the dead weight, which can ensure the loading accuracy during the measurement process, but most of them are produced of the 1970s. The loading system has the disadvantages of complicated wiring, large volume, single function and high failure rate, which brings many deficiencies and inconveniences to the hardness calibration work [3-5]. In the step of indentation measurement, the optical microscope is usually used, then people manually find the indentations and read the diameters of them, finally calculate the Brinell hardness value. These operations will probably involve personal measurement errors in results, and different operators might get different results for the same indentation. Moreover, measurement result may be different even when the same operator measures the same indentation multiple times. During the process, the operator is easy to get eye fatigue and it is more time-consuming to find smaller indentation points and the low efficiency is also the shortcoming of this traditional method [6][7].

Existing indentation measuring instruments include tool microscopes, image measuring instruments, and contact and non-contact geometry measuring instruments based on mechanical vision technology; first of all, these instruments are not dedicated Brinell hardness indentation measuring equipment, combining with the test force part of the machine it is difficult to realize automatic measurement; secondly, when measuring the indentation, the instruments need to move the reference block from the calibration machine to its measurement platform, this will inevitably introduce the influence of temperature [8]. Furthermore, when the diameter of the indentation is small, the instruments will be more timeconsuming when searching for the indentation.

At present, a new round of revolutions, such as machine vision, intelligent control, new energy and new materials, is emerging around the world. The rapid development of measurement and control technology and machine vision technology has made Brinell hardness measurement surely move towards automation [9][10]. Based on these two 
technologies and combining ISO6506-3:2014 and ISO6506-2:2017 developed a calibration machine as shown in Figure 1.

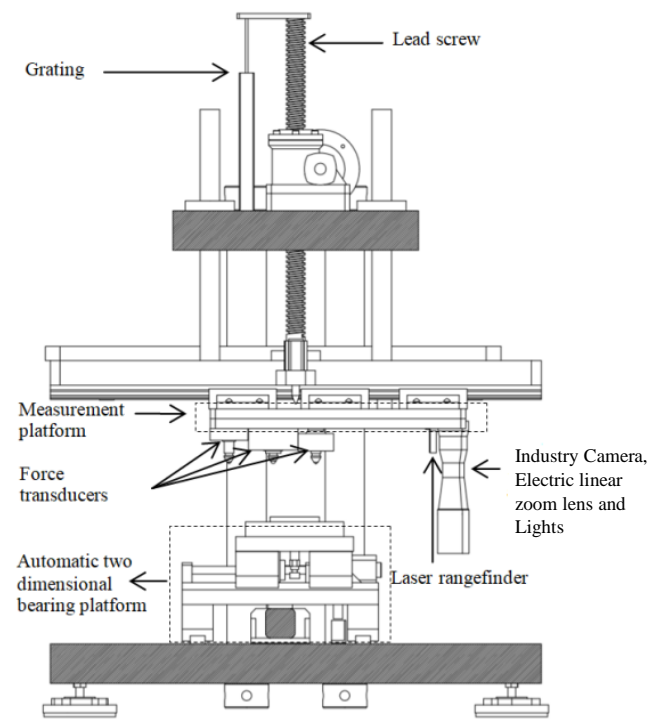

Figure 1: Calibration machine for reference block

The calibration machine is mainly composed of three parts: loading part, measuring part and twodimensional bearing platform. The loading part mainly includes the servo motor, reducer, lead screw, guide rod, measuring platform and upper and lower plates; the measuring part mainly consists of the force transducer group, laser rangefinder, indentation measurement system which are installed on the measuring platform and lead screw grating.

\section{DESIGN OF THE AUTOMATIC INDENTATION MEASUREMENT SYSTEM}

\subsection{Determine the Parameters of Core Components}

The indentation measurement system based on mechanical vision is mainly composed of industrial cameras, telecentric lenses, and light sources. The selection of industrial cameras and telecentric lenses is mainly determined based on the measured feature size and calibration accuracy requirements.

The main parameters of industrial cameras are resolution and sensor size; the main parameters of telecentric lenses are resolution, working distance, field of view area and magnification.

The optimal selection of the indentation system is that the size of the detected feature is equal to its field of view area, which is enlarged by the telecentric lens magnification to the size of the target surface of the industrial camera. In this case, the camera pixels will be fully used to characterize the measured indentation, thereby reducing the cost of the calibration device. According to the resolution calculation formula (1) of the industrial camera, the requirements of the detected feature and the measurement accuracy must be obtained.

$R=\frac{L_{\mathrm{MAX}}}{L_{\mathrm{MIN}}} \times p$

with $R$ - resolution;

$L_{\mathrm{MAX}}$ - detected feature;

$L_{\text {MIN }}$ - accuracy;

$p$ - It indicates that several pixels are used to characterize the measurement accuracy. (If the entire image is regarded as a periodic signal with a minimum feature size, according to Nyquist sampling law, the signal must be sampled at least 2 points per cycle to fully reproduce the signal.)

The measured feature size is determined according to ISO6506-1:2014 7.4, The test force should be chosen so that the diameter of the indentation, $\mathrm{d}$, lies between the values $0.24 \mathrm{D}$ and $0.6 \mathrm{D}$ [11], as there are four types of hard metal ball diameters: $10 \mathrm{~mm}, 5 \mathrm{~mm}, 2.5 \mathrm{~mm}$, and $1 \mathrm{~mm}$, the corresponding indentation diameter ranges are shown in Table 1. ISO6506-3:2014 4.6 shows the requirements for the measurement accuracy of the indentation measurement system in Table 1, [12]. Combine the indentation diameter range with the corresponding accuracy requirements to calculate the requirements for the resolution of industrial cameras.

Table 1: Indentation range and performance requirements of indentation measurement system

\begin{tabular}{|c|c|c|c|}
\hline $\begin{array}{c}\text { Hard metal } \\
\text { ball diameter } \\
(\mathrm{mm})\end{array}$ & $\begin{array}{c}\text { Indentation } \\
\text { Range } \\
(\mathrm{mm})\end{array}$ & $\begin{array}{c}\text { Diameter of } \\
\text { indentation } \\
(\mathrm{mm})\end{array}$ & $\begin{array}{c}\text { Performance } \\
(\mathrm{mm})\end{array}$ \\
\hline 10 & $2.4 \sim 6$ & $d \geq 2.5$ & \pm 0.0020 \\
\cline { 1 - 2 } & $1.2 \sim 3$ & \multirow{2}{*}{$1 \leq d<2.5$} & \pm 0.0010 \\
2.5 & $0.6 \sim 1.5$ & \multirow{2}{*}{$d<1$} & \pm 0.0005 \\
\hline 1 & $0.24 \sim 0.6$ & & \\
\hline
\end{tabular}

\subsection{Configuration of Automatic Indentation Measure System}

Because the range of indentation diameter is wide and the measure performance of different ranges is also different, if using a fixedmagnification telecentric lens with an ultra-highpixel industrial camera can realize the automatic measurement of various indentations. But in this 
configuration the indentation measurement system needs to ensure the complete shoot of the full maximum indentation diameter $(6 \mathrm{~mm})$, at the same time, it must also meet the measure accuracy requirement $(0.0005 \mathrm{~mm})$ of the minimum indentation diameter. When 2 pixels are used to characterize the measurement accuracy, according to the resolution calculation formula, $R$ is equal to 24000 , the camera resolution would be $24,000 \times 24,000$ about 576 million resolution. The camera cost will be extremely high. If one camera is used with multiple telecentric lenses, the hardware configuration cannot achieve automatic measurement of the full diameter range.

The use of the electric linear zoom lens will reduce the camera cost and achieve automatic measurement of the full diameter range. Firstly, the lens can be automatically adjusted to the specified magnification. When the lens enlarges a small indentation, the field of view will be reduced as well, so that the pixels can fully characterize the indentation.

The automatic indentation measuring system uses an electric linear zoom lens with built-in coaxial blue light and its lens field of view can be adjusted to $6 \mathrm{~mm}, 2.5 \mathrm{~mm}$ and $1 \mathrm{~mm}$, so each field of view can ensure the largest indentation can be sampled. At the same measurement accuracy condition, the larger indentation feature requires the higher resolution of the camera, so the camera resolution must meet the maximum indentation measuring requirement. When the indentation diameter range is between $2.5 \mathrm{~mm}$ and $6 \mathrm{~mm}$, the indentation measurement accuracy is required to be $0.002 \mathrm{~mm}$. When 2 pixels are used to characterize the measurement accuracy, calculated as follows according to the resolution formula (1):

$$
\begin{aligned}
R & =\frac{L_{\mathrm{MAX}}}{L_{\mathrm{MIN}}} \times p \\
R & =\frac{6 \mathrm{~mm}}{0.002 \mathrm{~mm}} \times 2 \\
R & =6000 .
\end{aligned}
$$

At this time, the maximum feature of the indentation is equal to the field of view. Based on the above conditions, the pixel requirement in one direction of the camera is calculated to be 6000 , since the detected indentation is a diameter, the number of pixels required in the other direction is also 6000, then ideally, the resolution of 36 million can meet the requirements of measurement accuracy. However, there are few industrial camera sensor on the market, generally rectangular with an aspect ratio of 1.33 , such as $12.8 \times 9.6,6.4 \times 4.8$, etc. Therefore, in order to facilitate camera selection, when the number of vertical pixels is 6000 , the number of horizontal pixels is $6000 \times 1.33$ is about 8000 , then the actual camera resolution selection is about 50 million. According to the above calculation process combined with the three field of view diameter and measurement accuracy requirements to calculate the corresponding resolution requirements, as shown in Table 2.

Table 2: Requirement of camera resolution

\begin{tabular}{|c|c|c|}
\hline $\begin{array}{c}\text { Diameter of } \\
\text { indentation } \\
(\mathrm{mm})\end{array}$ & $\begin{array}{c}\text { Performance } \\
(\mathrm{mm})\end{array}$ & $\begin{array}{c}\text { Required } \\
\text { camera } \\
\text { resolution }\end{array}$ \\
\hline$d \geq 2.5$ & \pm 0.0020 & 6000 \\
\hline $1 \leq d<2.5$ & \pm 0.0010 & 5000 \\
\hline$d<1$ & \pm 0.0005 & 4000 \\
\hline
\end{tabular}

According to Table 2 it can be found that when the measurement accuracy of the indentation is $0.002 \mathrm{~mm}$ and the field of view area is $6 \mathrm{~mm}$, the requirement of the camera resolution is the highest. It is concluded that the camera with about 50 million pixels and equipped with electric linear zoom lens can meet the automatically measurement and accuracy requirements.

\section{DESIGN THE FORCE PART OF THE CALIBRATION MACHINE}

The test force of this calibration machine is provided by high-precision force transducers, according to the ISO 6506-1: 2014-7.3, and the requirement in ISO 6506-3: 2014-4.4, selects ISO 376 class 0.03 high-precision force transducers and range of $30 \mathrm{kN}, 3 \mathrm{kN}$ and $300 \mathrm{~N}$ to be installed on the measurement platform. When the test condition is selected, the corresponding force transducer will move to the loading position, this process is implemented by the linear guide rail, slider, screw rod, grating and servo motor on the measurement platform.

When the force transducer at the loading position, the axis of the force transducer coincides with the axis of the loading screw to avoid the generation of lateral force, and the loading and unloading of the test force is then completed through the measurement and control system and the loading mechanism.

The two-dimensional bearing platform is composed of steel plate, screw, grating ruler, linear guide, slider and servo motor. The platform is precisely controlled by software to move the reference block. And write the requirements of 
ISO6506-3:2014 7.8 indentation selection into the software, so as to improve the efficiency of loading point selection and make better use of the test area of the reference block.

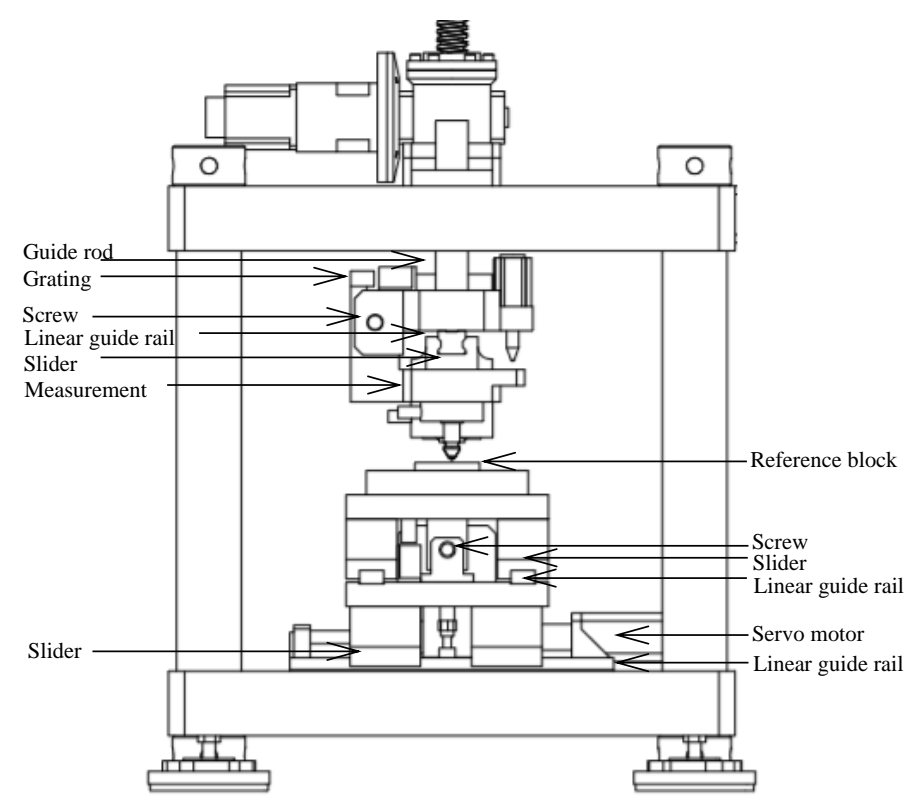

Figure 2: Main parts for moving the force transducer and two-dimensional bearing platform

\section{AUTOMATIC CALIBRATION PROCEDURE}

\subsection{The Key Point of Keeping Indentation Measurement Accuracy During The Calibration Procedure}

The principle of machine vision image measurement is to map the length in the world coordinate system to the pixels in the camera coordinate system in combination with the imaging system model. The better reproduction of the calibration conditions is most conducive to ensuring the accuracy of machine vision indentation measurement. Before the indentation measure system calibration, the camera should be installed vertically on the measurement platform to reduce the error caused by perspective distortion. The rest influence factors such as lens distortion and tangential distortion are caused by the hardware itself. Once the system calibration is completed, they are not easy to change during measurement. The calibration process is to map the real length to the indentation measurement system pixel and find its work distance. The work distance of the indentation test system is the most important condition to ensure accuracy, because the change of the distance will directly affect the mapping relationship between pixel points and world coordinates. In order to ensure the reproducibility of the work distance, the laser rangefinder, the grating of monitoring the lead screw combined with advanced calibration procedures to ensure this condition.

\subsection{Reference Block Calibration Procedure}

The first step is to place the reference block on the two-dimensional bearing platform, and then select the test force and light source according to the test condition.

The second step is to determine the longitudinal sample point of the indentation measurement system, due to the inconsistency of the reference block thickness and it has the surface problem of straightness and parallelism, so the indentation measurement system must also move longitudinally to ensure the reproducibility of the calibration distance. To solve the above problem, the hardware configuration and calibration methods are as follows:

a. The servo motor on the measuring platform moves the laser rangefinder to the horizontal working position through the linear guide, slider, screw and grating. In the working position, the optical point of the laser rangefinder coincides with the axis of the lead screw.

b. Then the laser rangefinder combined the thickness of the reference block preloading point with work distance of indentation measurement system find the longitudinal sample point, and the grating which monitors the lead screw records this longitudinal position. This position will be the longitudinal sample position of indentation measurement system, and then the measurement platform rises back to the initial position.

The third step, the force transducer corresponding to the test force is moved to the horizontal working position. In the working position, the axis of the force transducer coincides with the axis of the lead screw to avoid the generation of lateral force, and then the test force is applied through the measurement and control system and the loading mechanism. After completing the above operations, the measurement platform rises back to the initial position.

The fourth step: First, the indentation measurement system is moved to the horizontal working position. At this position, the centre of the lens coincides with the axis of the lead screw, and then the software combine the lead screw with its grating to move the indentation measurement system to the longitudinal sample position recorded in the second step $b$.

In the fifth step, the software processes the sampled image and calculates the hardness value. After the work is completed, the measurement 
platform and each measurement component are restored to the initial position, and then the next test point is automatically or manually selected according to the requirements by the twodimensional platform.

\section{SUMMARY}

This calibration machine has the following advantages: first, it completes the automatic calibration of the Brinell standard block through an advanced calibration program combined with the measurement and control system and the automatic indentation measurement system.

Second, due to the application of test force and the indentation are both on the two-dimensional pressure platform, this reduces the influence of the temperature by placing a certain number of reference blocks on the two-dimensional bearing platform in advance.

Third, according to the calibration procedure, the laser rangefinder, force transducer, and indentation measurement system will be moved to the same horizontal working position by the measurement platform during work, which ensures that the indentation is located in the centre of the field of view when sampling indentation, thus saving the time of finding the indentation and juxtapose it with the centre of the field of view so this improves the calibration efficiency.

When other geometry measuring instruments based on mechanical vision technology measured the reference block, the indentation is formed. Therefore, it can only use the surface other than the indentation to determine the working distance of the instrument and the reference block also has problems with straightness, parallelism and surface roughness, so it will affect the measurement results to a certain extent. Compared to the instruments, this calibration machine's work distance is obtained before the indentation formed. Therefore, the influence of the working distance on the measurement result can be reduced to a certain extent.

\section{REFERENCES}

[1] Yongchang Huang, Cheng Zhou, Tao Chen, Song Li, Guiping Qiu "Development of Brinell Hardness Indentation Automatic Measuring Device Based on High Definition Digital Image Analysis", Measurement Technique, pp. 10-14, July 2019.

[2] Wei Zhang, Xin Luo, Jiachun Lin, "Automatic control system for standard Brinell hardness machine based on PLC", Journal of Mechanical \& Electrical Engineering, pp. 211-212, Feb 2020.

[3] Jizeng Tao, Wei Shi, Jinying Li, Zhongyan Wu, "Application of Piezoelectric Ceramics to the Force Loading System of the Brinell Hardness Standard Machine”, Measurement \& Control Technology, pp. 72-73, Nov 2011.

[4] Yanan Yu, Wei Shi, Wu Zeng, Jizeng Tao, Ming $\mathrm{Xu}$, Jinying Li, "Development of electric Brinell hardness standard device", China Metrology, pp. 72-74, Sep 2010.

[5] Jintao Le, "Development Status and Direction for the Hardness Testing Machine Manufacturing Industry in China", Physical Testing and Chemical Analysis (Part A: Physical Testing), pp. 379-381, June 2017.

[6] Jizeng Tao, Wei Shi, Jinying Li, "The Brinell Hardness Measurement Device Based on CCD and Grating Ruler", Metrology \& Measurement Technology, pp. 18-20, June 2011.

[7] Wutingting Di, "Computer Measurement System of Brinell Hardness Indentation ", Digital Technology and Application, pp. 48+51, Nov 2016.

[8] Manlong Chen, "The fast calibration method for image measuring instrument", Modern Manufacturing Engineering, pp. 126-130+140, Aug 2017.

[9] Weiguang Lu, Guoyuan Wang, HaiLiang Wang, Zheng Cai "A Brief Talk on Geometric Measurement Technology and Its Application in Industrial Manufacturing Industry", Aviation Precision Manufacturing Technology, pp. 43-46, May 2019

[10] Yongchang Huang, Wei Huang, Bin Chen, Yu Bai, Mingtao Feng, Feiyu Zhao, "Application of Brinell Hardness Indentation Automatic Measuring Device in Verification", Metrology \& Measurement Technology, pp. 114-117, Jan 2016.

[11] ISO 6506-1:Metallic materials -Brinell hardness test-Part 1: Test method[s]. Switzerland Gevena: International Standardization Organization,2014

[12] ISO 6506-3:Metallic materials -Brinell hardness test-Part 3: Calibration of reference blocks[s]. Switzerland Gevena: International Standardization Organization,2014 